\title{
MAPA DE VULNERABILIDAD HIDROGEOLÓGICA DE UNA PARTE DEL VALLE CENTRAL DE COSTA RICA
}

\author{
Roberto Ramírez Ch. ${ }^{1}$ \& Alonso Alfaro M. ${ }^{2}$ \\ Servicio Nacional de Aguas Subterráneas, Riego y Avenamiento \\ SENARA Apdo. 5262 - San José \\ E-Mail:1 rramirezch@yahoo.com.mx \\ 2 ajesusa@yahoo.com
}

\begin{abstract}
This work presents a hydrogeological vulnerability map of a part of Valle Central in Costa Rica. The study area is characterized by a high density urban population, where the presence of industry and agriculture negatively impact the groundwater resources. The DI-O-S method was used to define the hydrogeological vulnerability, based in three factors: type of aquifer, lithology and depth of groundwater level. The zones with high vulnerability are located to the North and East sides of the Valle Central. These zones must be investigated in great detail to be considered in further urban management plans.

RESUMEN: Este trabajo presenta el mapa de vulnerabilidad hidrogeológica de una parte del Valle Central de Costa Rica, donde se concentra la mayor parte de la población del país, se desarrolla gran parte de la industria, agricultura y otros procesos productivos que impactan de forma negativa a los recursos subterráneos. Se utilizó el método de DI-O-S, que se basa en el producto de tres parámetros: ocurrencia del agua subterránea, sustrato litológico y profundidad del nivel de agua subterránea. Se puede observar que las áreas de mayor vulnerabilidad hidrogeológica se localizan al norte y este del Valle Central. Estas zonas, deben investigarse con mayor detalle, para ser consideradas en los planes de ordenamiento del territorio.
\end{abstract}

\section{INTRODUCCIÓN}

El Valle Central, ubicado en la parte central de Costa Rica, es ocupado por aproximadamente 2,5 millones de habitantes lo que representa un poco más del $50 \%$ de la población nacional. En él se concentra una gran parte de la actividad socioeconómica del país. Comprende las ciudades principales de las provincias de San José, Alajuela, Heredia y Cartago. Allí el crecimiento poblacional ha venido en aumento acompañado de una mayor demanda de servicios diversos que en muchos casos no han podido ser satisfechos. La expansión del desarrollo indus- trial, la demanda de zonas residenciales, entre otros, conduce a una acelerada alteración del ambiente natural.

Existen importantes zonas agrícolas y de desarrollo turístico, donde ya se ha iniciado un uso importante del recurso hídrico subterráneo y no hay conocimiento del régimen real de explotación ni del potencial de las fuentes subterráneas.

Durante las décadas pasadas se realizaron algunos estudios importantes sobre las aguas subterráneas, principalmente con fines de consumo humano en importantes centros de población, sin embargo, son estudios que no reflejan la situación actual y las tendencias de crecimiento. Algunos 
de esos estudios ofrecieron algunos resultados en la determinación de la disponibilidad de las aguas subterráneas -cuantificación de la cantidad-; algunos de ellos determinaron, también, su calidad. Tanto el AyA como el SENAS, y SENARA en los últimos dieciocho años, han realizado esfuerzos por investigar el recurso hídrico subterráneo; sin embargo, por lo general, el área de estudio era pequeña, no permitiendo hacer regionalizaciones completas.

Este trabajo tiene como objetivo elaborar el mapa de vulnerabilidad hidrogeológica de una parte del Valle Central de Costa Rica, usando la base topográfica, a escala 1: 200 000, para ser usado como una herramienta básica en la planificación regional del territorio.

El área comprende aproximadamente $1960 \mathrm{~km}^{2}$, entre las coordenadas 202-240 N y 496-545 E de la cuadrícula Lambert Costa Rica Norte, abarcando en gran parte lo que se conoce como Valle Central (Fig.1).

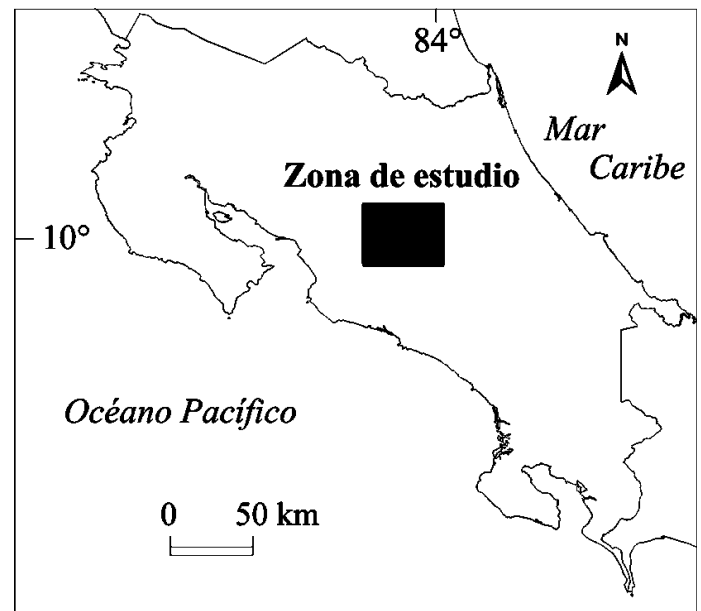

Fig. 1: Ubicación de la zona de estudio.

Para definir la vulnerabilidad se utilizó la metodología definida por Foster et al. (1992), que aparece en el documento "Estrategias Para la Protección de Aguas Subterráneas". La vulnerabilidad del acuífero, se define como un conjunto de características intrínsecas de los estratos geológicos, que separan la zona saturada del acuífero de la superficie del suelo y que determina la sensibilidad del acuífero a ser afectado adversamente por una carga contaminante aplicada.
Foster et al. (1992) definen las características de los estratos, en tres factores:

1) El grado de accesibilidad o grado de confinamiento de la zona saturada del acuífero a la penetración de contaminantes en un sentido hidráulico.

2) Las características generales de los estratos sobre la zona saturada en términos de litología y grado de confinamiento.

3) La profundidad del nivel freático o techo del acuífero confinado, que es una medida de:

a) Distancia que tiene que viajar la carga contaminante.

b) El tiempo que tardará para llegar hasta el acuífero.

c) Oportunidad para que los estratos atenúen el contaminante.

Para las asignaciones de usos del suelo, se utilizaron los manuales de Hirata et al. (1997), Foster et al. (1992), Vargas (1997) y Cruz (1997).

Para la elaboración de este mapa hidrogeológico, se recopiló toda la información hidrogeológica del Valle Central de Costa Rica, generada por el área de Aguas Subterráneas del SENARA y trabajos de consultores en Hidrogeología. Se digitalizó toda la información y se generaron diferentes coberturas en un Sistema de Información Geográfico (GIS).

Los mapas contemplados en el análisis son: mapas base a escala 1:200 000, con curvas de nivel cada $100 \mathrm{~m}$, pozos de Archivo Nacional (SENARA, 2002) y la base de datos de manantiales del Departamento de Aguas del MINAE, líneas isofreáticas (líneas de flujo) elaboradas por profesionales en hidrogeología del SENARA, A y A y consultores privados.

\section{CONTEXTO HIDROGEOLÓGICO DEL VALLE CENTRAL}

Los materiales geológicos que se encuentran en esta zona del país, tienen una edad de aproximadamente de 5 millones de años. Fueron originados por fracturas que permitieron la erupción de lavas y flujos piroclastos de las formaciones Grifo 
Alto y Doán, como también las coladas de las Lavas Intracañón y los depósitos de Avalancha Ardiente. Con la formación de los edificios volcánicos de la cordillera Volcánica Central, se dieron los depósitos de lahares y cenizas que rellenaron la depresión originada entre la cordillera y el volcanismo del Mioceno, todo este conjunto de procesos y materiales es conocido actualmente como Valle Central (Denyer et al., 1994). Está formación geológica del Valle Central, origina también los sistemas de acuíferos Colimas y Barva

Para empezar a estudiar estos acuíferos, se aclara que la momenclatura hidrogeológica y geológica no son iguales, porque fueron propuestas para diferentes objetivos. Se pueden establecer las siguientes semejanzas: las Lavas Intracañón, corresponden con la Formación Hidrogeológica Colima (Superior e Inferior), la formación Avalancha Ardiente correspondería a Tiribí y los piroclastos y lavas de los estravolcanes con la formación hidrogeológica Barva. Está forma de relacionar las formaciones geológicas con las hidrogeológicas se pueden observar mejor en la columna de la figura 2. (SENARA-BGS, 1985 y Arredondo, 1994).

\section{FORMACIÓN HIDROGEOLÓGICA COLIMA (LAVAS INTRACAÑÓN)}

Echandi (1981) le denomina Formación Colima e incluye los miembros Belén, Ignimbri- ta Puente de Mulas y Linda Vista. Identifica aproximadamente 7 coladas de lavas andesíticas de 10 a 30 metros de espesor que afloran solamente en los profundos valles de los ríos Virilla y Tiribí, pero que han sido encontradas en muchas de las perforaciones que se han realizado en el Valle Central. Localmente presenta una intercalación de una toba de flujo brechosa (Miembro Puente de Mulas) con un espesor máximo de 35 m. El espesor total de la formación es de casi $100 \mathrm{~m}$, y su volumen de por lo menos $35 \mathrm{~km}^{3}$ (Echandi, 1981). Uno de los tres miembros hidrogeológicamente diferenciables es el Miembro Linda Vista, que se encuentra extendido en gran parte del Valle Central. Aflora en la margen derecha del río Virilla, en los manantiales de Guachipelín y túneles de Puente Mulas. Formado por dos capas lávicas de composición andesítica, de textura brechosa o masiva fracturada, separados por una capa fina de toba meteorizada (10 cm de espesor aprox.) que actúa como acuitardo. La capa superior posee unos $110 \mathrm{~m}$ de espesor promedio en el que se ubica el acuífero colgado La Libertad y origina manantiales de 100 1/s (Fig 3), éste acuífero da origen a los manantiales del mismo nombre en el cañón del río Virilla, son lavas y brechas con un espesor promedio entre 75 y $90 \mathrm{~m}$. En el cuadro 1 se presentan de forma general las características hidráulicas de este acuífero.

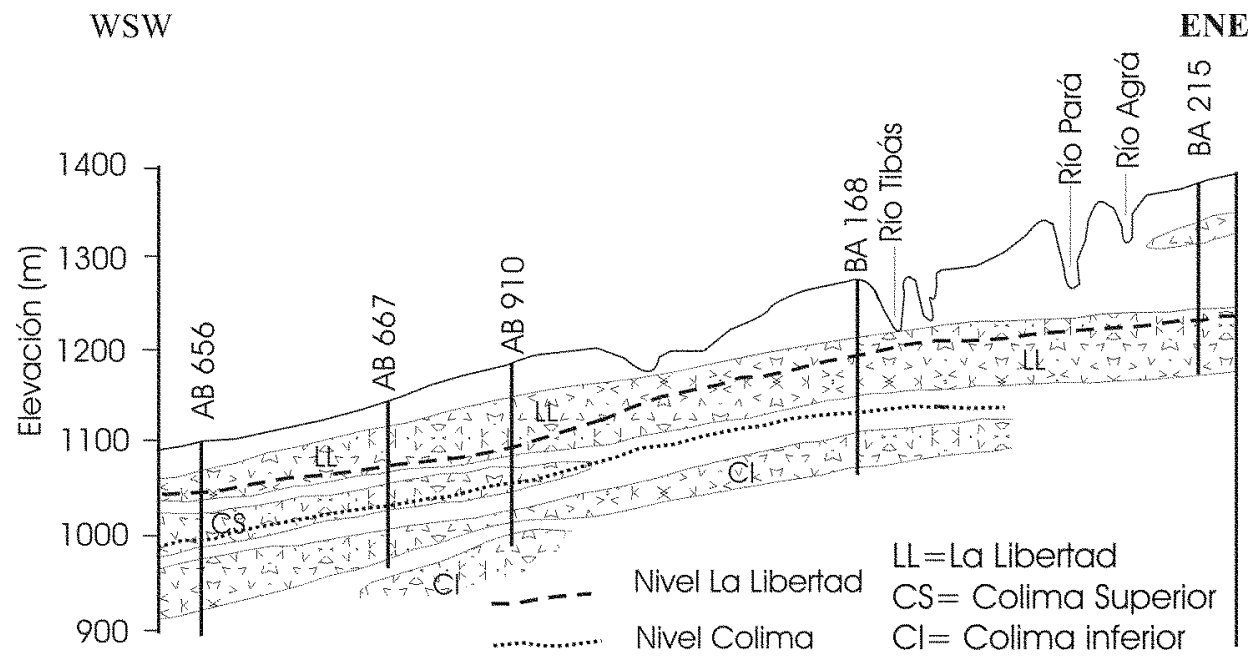

Fig. 2: Columna hidrogeológica del Valle Central (SENARA-BGS, 1985 y Arredondo, 1994). 


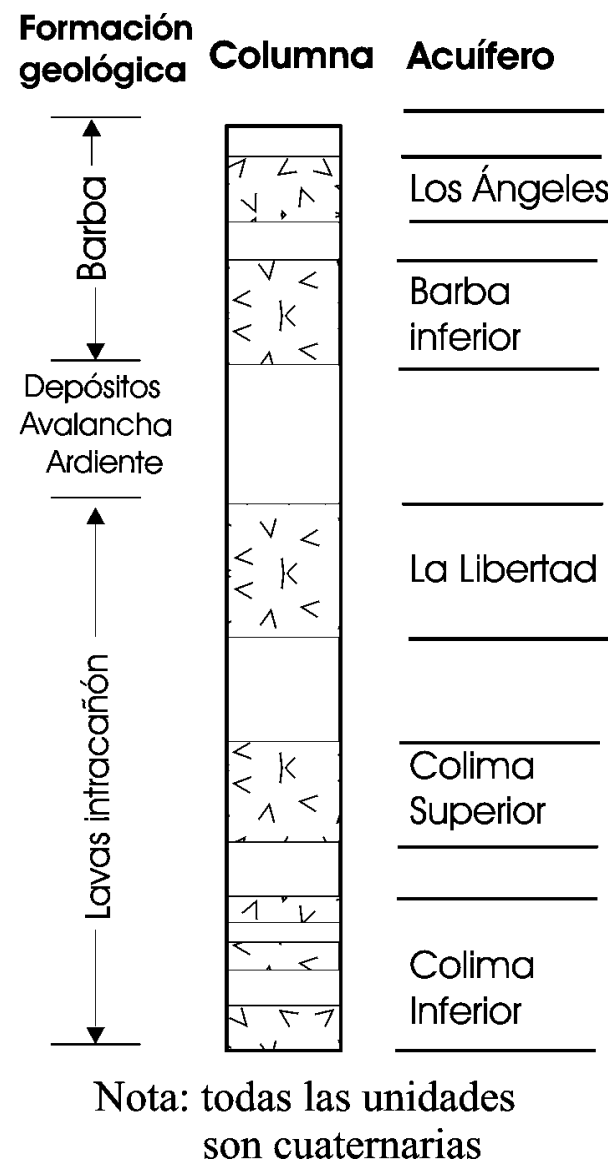

Fig. 3: Perfil hidrogeológico del Valle Central, cerca del campo de pozos la Valencia (SENARA-BGS, 1988).

Cuadro 1

Características hidráulicas de la formación hidrogeológica Colima

\begin{tabular}{ll}
\hline Parámetro Hidráulico & Valores \\
\hline Tipo de litología & $\begin{array}{l}\text { Lavas fracturadas e intercaladas } \\
\text { con capas piroclásticas: tipo } \\
\text { acuitardo o acuicierre. }\end{array}$ \\
Espesor Saturado & $>100 \mathrm{~m}$ \\
$\begin{array}{l}\text { Transmisividad } \\
\text { Coeficiente de } \\
\text { almacenamiento }\end{array}$ & 500 o mayores a $5000 \mathrm{~m}^{2} /$ día \\
Producción por pozo & $50-1001 / \mathrm{s}$ o mayores \\
\hline
\end{tabular}

Actualmente, el acuífero Colima se explota por parte de Acueductos y Alcantarillados de la siguiente forma: $1280 \mathrm{l} / \mathrm{s}$ del campo de pozos La Valencia, 650 1/s del manantial de Puente de Mulas, 250 1/s de Potrerillos y más de 400 litros de pozos y fuentes privadas.

\section{FORMACIÓN TIRIBÍ (DEPÓSITOS DE AVALANCHA ARDIENTE)}

Denominada Formación Tiribí por Echandi (1981) $y$ subdividida en los miembros Nuestro Amo, Electriona y La Caja. Krushensky (1972) le describe como una serie ampliamente distribuida en la que predominan depósitos de nubes ardientes (flujos de ceniza, flujos de pómez, flujos de bloques, lapilli y ceniza e ignimbritas)

Los flujos piroclásticos tienen un espesor promedio de 45 a $150 \mathrm{~m}$, su extensión longitudinal sobrepasa los $40 \mathrm{~km}$ al N y NW del Valle Central. Cubren un área de $500 \mathrm{~km}^{2}$ y tiene un volumen de $25 \mathrm{~km}^{3}$. Su forma es bastante tabular y se acuña hacia el este. El espesor de los flujos piroclásticos, el diámetro de las bombas escoriáceas y la frecuencia de las facies soldadas aumenta hacia el oeste, lo cual indica que no pueden provenir de los volcanes de la Cordillera Central (Denyer \& Arias, 1991; Denyer et al. 1994).

Desde el punto de vista hidrogeológico está formación se considera como un acuitardo y según algunos autores como acuicierre y se le asignan valores de permeabilidad entre 1,16-2,72 * $10^{-4}$ metros por día.

\section{FORMACIÓN HIDROGEOLÓGICA BARVA}

Incluye varias coladas y depósitos piroclásticos que se originaron en la cordillera Volcánica Central, forman parte del Grupo Volcánico Central y han sido incluidas dentro de la Formación Lavas Post-Avalancha y otros nombres usados para las coladas más meridionales, como la Colada de Cebadilla, la cual se presenta en forma de promontorios de bloques que sobresalen de la planicie ignimbrítica. Está constituida por coladas de 
lavas andesíticas y andesito-basálticas con espesores que van desde 10 hasta $80 \mathrm{~m}$ con intercalaciones de aproximadamente $10 \mathrm{~m}$ de cenizas y ocasionalmente lapilli (Denyer \& Arias, 1991).

Se dividide en cuatro miembros hidrogeológicamente bien diferenciables (Echandi, 1981; Arredondo, 1995): Miembro Bermúdez, que son lavas andesíticas fracturadas; Miembro Porrosatí y Carbonal, constituido por arenas volcánicas gruesas y tobas arcillosas meteorizadas, que forman acuitardos; Miembro los Ángeles y los Bambinos que comprende coladas de lava superiores que forman acuíferos discontinuos, colgados y de poca extensión. La recarga es debida a la infiltración de lluvia en las partes altas del volcán Barva y en los lechos de los ríos. Son comunes los manantiales en la base. Por último el Miembro Cráter, constituido por piroclástos recientes, que cubren parcialmente el acuífero de Barva Superior. Presenta espesores menores de $10 \mathrm{~m}$ y una capacidad moderada de infiltración. Las principales características hidráulicas de la Formación Barva descritas por Gómez (1987) se presentan en el cuadro 2.

\section{MAPA DE VULNERABILIDAD DE UNA PARTE DEL VALLE CENTRAL}

El método utilizado es el "DI-O-S" DI-distancia al agua, O-ocurrencia de agua subterráneas

Cuadro 2

Características hidráulicas de la formación hidrogeológica Barba

\begin{tabular}{ll}
\hline Parámetro Hidráulico & Valores \\
\hline Tipo de litología & $\begin{array}{l}\text { Lavas y tobas de aparente } \\
\text { permeabilidad baja }\end{array}$ \\
$\begin{array}{l}\text { Permeabilidad } \\
\text { (metros por día) }\end{array}$ & $1 \mathrm{~m} / \mathrm{d}-10 \mathrm{~m} / \mathrm{d}$ \\
$\begin{array}{l}\text { Espesor saturado } \\
\text { Transmisividad }\end{array}$ & $100-50 \mathrm{~m}$ en promedio \\
$\begin{array}{l}\text { Coeficiente de } \\
\text { Almacenamiento }\end{array}$ & 0,1 \\
Producción de \\
manantiales
\end{tabular}

y S-sustrato geológico; se basa en una asignación de valores numéricos relativos. El primer valor se relaciona con el tipo de ocurrencia de aguas subterráneas (confinado o libre), dentro de un rango de $0-1$. El segundo es la caracterización de los estratos encima de la zona saturada, que tiene relación con el grado de confinamiento y el carácter litológico e indirectamente toma en cuenta, la porosidad relativa, permeabilidad y contenido de humedad o retención específica de la zona no saturada, tiene valores de 0,4-1,0. El último parámetro es la determinación de la profundidad del agua o techo para acuíferos confinados; el rango de valores es 0,4-1,0. Estos valores se multiplican y se obtiene un valor numérico que varía de $0-1,0$ y se asignan diferentes grados de vulnerabilidad: ninguna, baja, moderada, alta y extrema.

Por la complejidad acuífera del Valle Central, se tienen que simplificar las variables de vulnerabilidad, considerándose tres niveles de vulnerabilidad: baja, media y alta. Se utilizó una matriz sencilla, propuesta por Cruz (1997) y modificada por los autores para el Valle Central (Cuadro 3).

El mapa de vulnerabilidad hidrogeológica de una parte del Valle Central se dividió en tres zonas de vulnerabilidad, para cada una se recomiendan las limitaciones de uso (Cuadro 4).

Se puede observar una zona de alta vulnerabilidad en la parte norte (Fig.4), debido a los depósitos piroclásticos permeables, como también lavas fracturadas, que aumentan el grado de incidencia de los contaminantes. La vulnerabilidad media se localiza en las ciudades de Heredia, Santo Domingo, San Pablo, San Antonio y San Juan de Tibás; estas zonas poseen coberturas de tobas de muy baja permeabilidad. Las zonas de vulnerabilidad baja se caracterizan por espesores importantes y permeabilidades bajas de las rocas aflorantes.

\section{LIMITACIONES DEL MAPA}

Esta zonificación permite orientar recursos a estudios hidrogeológicos y complementarios como perforación, ensayos geofísicos y otros proyectos con el objetivo de desarrollar posibles 
Cuadro 3

Matriz de vulnerabilidad hidrogeológica

\begin{tabular}{lccc}
\hline Categoría / Parámetros & BAJA & MEDIA & ALTA \\
\hline DI- Distancia al Agua & $>30$ & $10-30$ & $<30$ \\
$\begin{array}{l}\text { O- Ocurrencia del } \\
\text { agua Subterránea }\end{array}$ & - & - & $\begin{array}{c}\text { Acuífero libre } \\
\text { (no confinado) }\end{array}$ \\
S- Sustrato litológico & $\begin{array}{c}\text { Unidad consolidada, poco } \\
\text { fracturada de baja permeabilidad, } \\
\text { (piroclastos, tobas y lahares) }\end{array}$ & $\begin{array}{c}\text { Piroclastos semiconsolidados } \\
\text { poco fracturados, con } \\
\text { permeabilidad media }\end{array}$ & $\begin{array}{c}\text { Piroclastos y lavas } \\
\text { fracturadas de alta } \\
\text { permeabilidad }\end{array}$ \\
\hline
\end{tabular}

Cuadro 4

Asignación de uso para diferentes grados de vulnerabilidad

\begin{tabular}{|c|c|}
\hline \multicolumn{2}{|r|}{ Zonas de vulnerabilidad alta } \\
\hline Urbanización & $\begin{array}{l}\text { Se permiten proyectos de baja densidad de población, 1-60 per/ha. Para reducir la contaminación } \\
\text { de cuerpos de agua e impermeabilización del suelo deben diseñarse las zonas de protección de } \\
\text { manantiales y pozos de abastecimiento de agua. }\end{array}$ \\
\hline Agricultura & $\begin{array}{l}\text { No permitir plantaciones de flores y plantas ornamentales. Incentivar las prácticas de agricultura } \\
\text { conservacionista; así como la eficiencia en la aplicación de los agroquímicos y realizar tratamiento } \\
\text { de los efluentes químicos y biodegradables. }\end{array}$ \\
\hline Industria & $\begin{array}{l}\text { No permitir la construcción de industrias peligrosas y medianamente peligrosas. Solo se permite } \\
\text { industrias inofensivas con sistemas de tratamiento de efluentes y desechos }\end{array}$ \\
\hline Rellenos sanitarios & No permitidos \\
\hline Canteras & $\begin{array}{l}\text { Realizar estudios hidrogeológicos detallados para no destapar el acuífero y exponerlo a cargas } \\
\text { contaminantes }\end{array}$ \\
\hline Turismo & $\begin{array}{l}\text { Se permiten proyectos de baja densidad de población turística con plantas de tratamiento de las } \\
\text { aguas residuales y una eficiencia en el uso del agua, realizar estudios hidrogeológicos detallados. }\end{array}$ \\
\hline \multicolumn{2}{|r|}{ Zonas de vulnerabilidad media } \\
\hline Urbanización & $\begin{array}{l}\text { Se permiten urbanizaciones de densidades medias, } 70-249 \text { personas por hectárea, con sistemas de } \\
\text { alcantarillado y planta de tratamiento. Deben diseñarse las zonas de protección de manantiales y } \\
\text { pozos de abastecimiento público. }\end{array}$ \\
\hline Agricultura & Permitida pero con estrictas regulaciones de la descarga y aplicación de agroquímicos y pesticidas. \\
\hline Industria & $\begin{array}{l}\text { Se permiten industrias medianamente peligrosas e inofensivas con sistemas de tratamiento de } \\
\text { efluentes y desechos sólidos }\end{array}$ \\
\hline Rellenos sanitarios & Permitidos con estudios hidrogeológicos detallados y tratamiento de los efluentes \\
\hline Canteras & $\begin{array}{l}\text { Se permiten pero se deben de realizar estudios hidrogeológicos detallados para no destapar los } \\
\text { acuíferos y exponerlos a cargas contaminantes. }\end{array}$ \\
\hline Turismo & $\begin{array}{l}\text { Permitidos proyectos de baja densidad turística, con plantas de tratamiento de aguas residuales y } \\
\text { promover una eficiencia en el uso del agua, realizar estudios hidrogeológicos detallados. }\end{array}$ \\
\hline \multicolumn{2}{|r|}{ Zonas de vulnerabilidad baja } \\
\hline Urbanización & $\begin{array}{l}\text { Se permiten urbanizaciones de densidades medias, } 70-249 \text { o más personas por hectárea, con } \\
\text { sistemas de alcantarillado y planta de tratamiento, diseñar las zonas de protección de manantiales y } \\
\text { pozos de abastecimiento público }\end{array}$ \\
\hline Agricultura & Permitidas pero con estrictas regulaciones de la descarga y aplicación de agroquímicos y pesticidas. \\
\hline Industria & $\begin{array}{l}\text { Se permiten industrias medianamente peligrosas e inofensivas con sistemas de tratamiento de } \\
\text { efluentes y desechos sólidos. }\end{array}$ \\
\hline Rellenos sanitarios & Permitidos con estudios hidrogeológicos detallados y tratamiento de los efluentes \\
\hline Canteras & $\begin{array}{l}\text { Se permiten pero se deben de realizar estudios hidrogeológicos detallados para no destapar los } \\
\text { acuíferos y exponerlos a cargas contaminantes. }\end{array}$ \\
\hline Turismo & Se recomienda sistemas de tratamiento de efluentes. \\
\hline
\end{tabular}




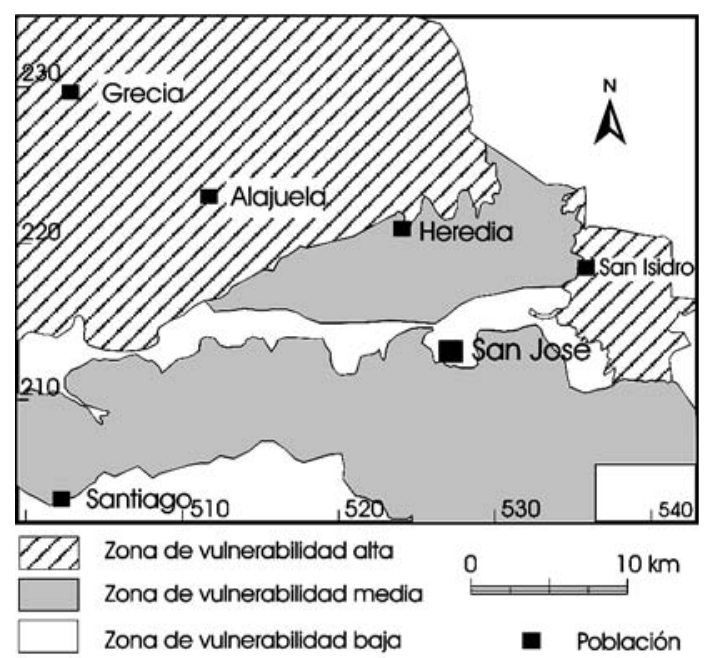

Fig. 4: Mapa de vulnerabilidad hidrogeológica de una parte del Valle Central.

fuentes de abastecimiento de agua potable como medidas y estrategias de protección.

La zonificación de vulnerabilidad bajo ninguna circunstancia sustituye los estudios hidrogeológicos locales, destinados al desarrollo de los recursos hídricos, como acueductos, captaciones, pozos y zonas de recarga locales.

\section{CONCLUSIONES Y RECOMENDACIONES}

La fuerte actividad urbana, agrícola, industrial y turística que se ha instalado en el Valle Central, ha creado la necesidad de plantear zonificaciones que tengan como objetivo la protección de los recursos hídricos, tanto subterráneos como superficiales.

Para este análisis se utilizó una metodología relativamente sencilla, que consta de tres parámetros: ocurrencia del agua subterránea, sustrato litológico y profundidad del nivel de agua subterránea. Se considera que para utilizar otro método se requiere de mucha información, que en este momento no se ha generado.

El mapa generado permite orientar investigaciones futuras de aguas subterráneas y debe ser usado como una herramienta básica en el ordenamiento del territorio de forma regional.
Según el mapa propuesto, la zona norte y este del Valle Central es considerada como de vulnerabilidad alta, probablemente coinciden con zonas de recarga acuífera; sin embargo, para su verdadera valoración deben realizarse estudios de detalle, que contemplen la geología a una escala adecuada, perforaciones exploratorias, balances hídricos, hidroquímica, pruebas de bombeo, medidas hidráulicas en profundidad y otras.

\section{REFERENCIAS}

ARREDONDO, S., 1994: Aguas subterráneas y fuentes termales. - En: Denyer, P. \& Kussmaul, S. (compiladores): Atlas geológico de la Gran Área Metropolitana, Costa Rica. - Edit. Tecnológica de Costa Rica: 197-210 pags.

ARREDONDO, S., 1995: Delimitación de la zonas de protección a los acuíferos en el área de influencia de Belén, Heredia. - 200 págs. SENARA, San José [Inf. interno].

CRUZ, M. O. A. ; 1997: Modelaje del acuífero Managua y su rendimiento sostenible. - 146 págs. Univ. de Costa Rica, San José [Tesis MsC.].

DENYER, P. \& ARIAS, O., 1991: Estratigrafía de la región central de Costa Rica. - Rev. Geól. América Central, 12: $1-59$.

DENYER, P., AGUILAR, T. \& ARIAS, O., 1994: Historia geológica. - En: Denyer, P. \& Kussmaul, S. (compiladores): Atlas geológico de la Gran Área Metropolitana, Costa Rica. - Edit. Tecnológica de Costa Rica: 98-108.

DENYER, P., KUSSMAUL, S. \& ARIAS, O., 1994: Estratigrafía de las rocas ígneas. - En: Denyer, P. \& Kussmaul, S. (compiladores): Atlas geológico de la Gran Área Metropolitana, Costa Rica. - Edit. Tecnológica de Costa Rica: 161-70.

ECHANDI, E., 1981: Unidades volcánicas de la vertiente norte del río Virilla. - 123 págs. Univ. de Costa Rica, San José [Tesis de Lic.].

FOSTER, S., BRIAN, A., MORALES, M. \& TENOJO, S., 1992: Estrategias de protección de aguas subterráneas, una guía para su implementación. - 91 págs. Centro Panamericano de Ingeniería Sanitaria y Ciencias del Ambiente (CEPIS).

GÓMEZ, A., 1987: Evaluación del potencial de los acuíferos y diseño de las captaciones de aguas subterráneas en la zona de puente de Mulas, Heredia, Costa Rica. Univ. de Costa Rica, San José [Tesis Lic.]. 
HIRATA, R., RIBEIRO de ASIS, C. \& ROCHA, G. De ALBUQUERQUE, 1997: Mapeamiento da vulnerabilidade e risco de poluicão dos aguas subterrãea no Estado de São Paulo. - Volumen I, 130 págs. Inst. Geol. CETESB-DAEE.

KRUSHENSKY, R.D., 1972: Geology of the Istaru Quadrangle, Costa Rica. - 46 pags. Bull. Geol. Surv. 1358:

SENARA, BGS \& AyA, 1988: Continuación de la investigación hidrogeológica de la zona norte y este del Valle Central. - 150 págs. SENARA, 165 [Inf. interno].
SENARA \& BGS, 1985: Mapa hidrogeológico del Valle Central de Costa Rica, escala 1:50000.

SENARA, 2002: Archivo nacional de pozos del área de aguas subterráneas del SENARA.

VARGAS, A., 1997: Ventajas del proceso de reforestación sobre la atenuación de la erosión y propuesta de protección y desarrollo de un área de recarga en el Valle Central de Costa Rica. - Rev. Geol. América Central, 19/20: $155-165$. 